\title{
A study on effect of glycemic control on cardiac autonomic function tests in type 2 diabetes
}

\author{
Padmanabha BV*, Karthiyanee Kutty, Srinivas Rao. \\ 1.Asst professor, Dept of Physiology, Faculty of Medicine, Jazan University. \\ 2.Professor, Dept of Physiology, SDUMC, Kolar. \\ 3.Professor, Dept of Medicine, SDUMC, Kolar.
}

\section{Abstract}

The present study was undertaken to estimate the glycosylated $\mathrm{Hb}$ level in type-2 diabetic subjects by cation-exchange- resin method and to study the relation between glycemic control and cardiac autonomic function. The study comprised of 90 subjects, 60 Diabetic mellitus type 2 patients and 30 age matched normal individuals. Diabetic mellitus type 2 patients were further classified in to two sub-groups, Type2 Diabetic mellitus patients with good glycemic control $(\mathrm{HbA} 1 \mathrm{c}<7)$ and Type 2 Diabetic mellitus patients with poor glycemic control $(\mathrm{HbA} 1 \mathrm{c}>7)$. The basis of finding is multifactorial and may be at multiple levels of neuraxis including peripheral and central mechanisms as reviewed under pathophysiology of diabetic autonomic neuropathy.

Key words: Cation-exchange- resin method, glycosylated $\mathrm{Hb}$, type-2 diabetes, cardiac autonomic function.

\section{INTRODUCTION}

Diabetes mellitus type 2 is a metabolic disorder primarily characterized by insulin resistance, relative insulin deficiency and hyperglycemia. Complications of Diabetes are the major cause of morbidity and mortality in persons with type 2 Diabetes mellitus.Chronic hyperglycemia is a major initiator of micro vascular complications of Diabetes. Micro vascular complications comprise Neuropathy, Retinopathy and nephropathy. Neuropathy includes both peripheral and autonomic neuropathy. Autonomic neuropathy is the most common complication of Diabetes, which mainly affects the cardiovascular system, gastrointestinal system and urogenital system. Cardiovascular autonomic neuropathy is the earliest form of autonomic dysfunction, which causes abnormalities in heart rate control as well as defects in central and peripheral vascular dynamics. Poor glycemic control plays an important role in the development and progression of diabetic cardiac autonomic neuropathy, and studies have shown that reduced cardiovascular autonomic function is associated with increased morbidity and mortality.

Researches in this regard have suggested a strong association between hyperglycemia and the progression of micro vascular complications in diabetic patients. Glycosylated hemoglobin reflects long-term glycemic control and has proven to be a more accurate and stable measure than fasting blood glucose levels. Quantitative cardiovascular autonomic function tests are widely used to detect, verify and quantify the cardiovascular autonomic dysfunction. They have been tested for their validity and reliability. These tests are performed because the procedures are straightforward, reproducible and non invasive. Earlier studies have suggested that patients with poor glycemic control have higher prevalence of cardiovascular autonomic neuropathy than in patients with good glycemic control in type 1 diabetes. Similar studies in type 2 Diabetes mellitus are relatively few. So this study is designed to evaluate

\footnotetext{
Address for correspondence*

Padmanabha BV

Asst professor,

Dept of Physiology, Faculty of Medicine,

Jazan University.
}

out the function assessed by cardiac autonomic function tests in Diabetes mellitus type 2 with poor control and compare it with Diabetes mellitus type 2 with good glycemic control and with age matched control group. Glycosylated hemoglobin levels are used as a measure of glycemic control.[1-20]

The present study was undertaken to estimate the glycosylated $\mathrm{Hb}$ level in type- 2 diabetic subjects by cationexchange- resin method and to study the relation between glycemic control and cardiac autonomic function.

\section{MATERIALS AND METHODS SUBJECTS}

The study comprised of 90 subjects, 60 Diabetic mellitus type 2 patients and 30 age matched normal individuals. Diabetic mellitus type 2 patients were further classified in to two subgroups, Type2 Diabetic mellitus patients with good glycemic control $(\mathrm{HbA} 1 \mathrm{c}<7)$ and Type2 Diabetic mellitus patients with poor glycemic control (HbAlc $>7)$. Informed consent was obtained from each subject. The study was approved by the Ethical committee, Sri Devaraj Urs Medical College, Kolar. After filling the questionnaire part of the pro-forma by the subjects, each study group was constituted as per the following criteria.Type2 Diabetic mellitus patients with good glycemic control: 30 subjects attending R.L.Jalappa Hospital who fulfilled the following criteria were included in the study. The glycosylated $\mathrm{Hb}$ level is estimated by cat-ion exchange resin method.

Inclusion criteria: The subjects should be type 2 diabetic diagnosed irrespective of duration of diabetes within the age group of 40-60 years, HbA1c level should be lesser than 7\%, Subjects should be a non smoker and non alcoholic, Subjects should not be suffering from any cardiovascular diseases, Subjects should not be on drugs altering autonomic functions.

Exclusion criteria: The subjects who are non- diabetic, HbA1c level more than 7\%, Subjects who are smoker and alcoholic, Subjects suffering from any cardiovascular diseases, Subjects on drugs altering autonomic functions.

Type2 Diabetic mellitus patients with poor glycemic control: 30 subjects attending R.L.Jalappa Hospital who fulfilled the following criteria were included in the study. The presence of diabetes was detected by random plasma glucose $>11.1 \mathrm{mmol} / \mathrm{L}$ (200 mg/dl)2sd. The glycosylated $\mathrm{Hb}$ level is estimated by cat-ion 
exchange resin method.

Inclusion criteria: The subjects should be type 2 diabetics irrespective of duration of diabetes within the age group of 40-60 years, HbA1c level should be greater than $7 \%$., should be a non smoker and non alcoholic, should not be suffering from any cardiovascular diseases, should not be on drugs altering autonomic functions.

Exclusion criteria: Subjects who are non-diabetic, HbAlc level less than 7\%., subjects who are smoker and alcoholic, subjects suffering from any cardiovascular diseases, subjects on drugs altering autonomic functions.

Control group: The control group consisted of 30 healthy subjects who were volunteers. They were chosen according to the following criteria.

Inclusion criteria: The subjects who are non-diabetic, HbA1c level should be lesser than $6 \%$., Subjects should be a non smoker and non alcoholic, Subjects should not be suffering from any cardiovascular diseases, Subjects should not be on drugs altering autonomic functions.

Exclusion criteria: The subjects who are diabetic, HbA1c level more than 6\%., Subjects who are smoker and alcoholic, Subjects suffering from any cardiovascular diseases, Subjects on drugs altering autonomic functions.

\section{METHODS}

The subjects were instructed not to have coffee, tea cola 12 hours before the tests and were asked to have light breakfast two hours before the tests. The subject was asked to relax in supine position for 30 minutes. The resting heart rate was recorded on a standard ECG from lead two, at a paper speed of 25 $\mathrm{mm} / \mathrm{sec}$. BP was measured with sphygmomanometer by the standard auscultatory RivaRocci method. The cardiovascular tests performed are detailed below in the order of execution. These tests were demonstrated to the subjects.

Procedure of autonomic evaluation as described by Ewing \& Clarke(1982)

Deep breathing test: In the sitting position subject was asked to breathe quietly and deeply at the rate of 6 breaths per minute. A continuous ECG was recorded for six cycles with marker to indicate the onset of each inspiration and expiration. The maximum and minimum R-R intervals were measured during each breathing cycles and converted to beats per minute. The result was then expressed as mean of the difference between maximum and minimum heart rate for six measured cycles in beats per minute.

Deep breathing difference $(\mathrm{DBD})=$ mean of heart rate differences in 6 breath cycles. A normal response was a difference of 15 beats/min or more, 11-14 beats/min borderline and less than 10 beats/min was considered abnormal.

Heart-Rate variation to Valsalva Maneuver: The subject was seated comfortably and was asked to blow into a mouthpiece connected to a mercury sphygmomanometer and holding it at a pressure of $40 \mathrm{~mm}$ of mercury for 15 seconds., while a continuous ECG was being recorded. The ECG was continued to be recorded after release of pressure at the end of 15 seconds for 30 seconds. The heart rate changes induced by the valsalva manoeuver was expressed as the ratio of the maximal tachycardia during the maneuver to the maximal bradycardia after the maneuver. This ratio was defined as the Valsalva ratio and was calculated as the ratio of maximum R-R interval after the maneuver to minimum $\mathrm{R}-\mathrm{R}$ interval during the maneuver. Valsalva ratio( $\mathrm{VR})=$

maximal tachycardia/maximum bradycardia $=$ maximum $\mathrm{R}-\mathrm{R}$ interval/minimum $\mathrm{R}-\mathrm{R}$ interval.A value of 1.10 or less is defined as an abnormal response, 1.11-1.20 as borderline, and 1.21 or more as a normal response.

Heart rate response to standing (postural tachycardia index): The subjects were asked to lie on the examination table quietly while heart rate is being recorded on ECG. They were then asked to stand-up unaided and ECG was recorded for 1 minute. The shortest R-R interval at or around 15th beat and longest R-R interval at or around 30th beat was measured. The result was expressed as ratio of 30/1. PTI= Longest R-R interval at 30th beat / shortest R-R at 15 th beat. A ratio of 1.00 or less was defined as an abnormal response, 1.01-1.03 as borderline and 1.04 as normal response.

Blood Pressure Response To Standing (Orthostatic test): The subject was asked to rest in a supine position for 5 minutes. The resting BP was recorded. The subject was then asked to stand unaided and remain standing unsupported for 3 minutes. The BP was recorded at 30 seconds and 3 minutes after standing up. The difference between the resting and standing BP levels was calculated. The fall in systolic BP at 30 seconds on standing noted. A fall of $30 \mathrm{~mm} \mathrm{Hg}$ or more was defined as abnormal, fall between 11-29 $\mathrm{mm} \mathrm{Hg}$ as borderline and fall of $10 \mathrm{~mm} \mathrm{Hg}$ or less was considered normal.

Blood Pressure Response To Sustained Handgrip: A blood pressure cuff was inflated and basal reading in the manometer was kept at $10 \mathrm{~mm}$ of mercury. The patient was then asked to compress the cuff to the maximum possible extent with one hand (as if squeezing a tennis ball) and reading on manometer was taken. This reading gives the maximum voluntary contraction. The patient was asked to maintain the pressure of cuff in such a way as to keep the manometer reading at $30 \%$ of maximum force for a period of 3 minutes. Blood pressure was recorded in the non exercising arm thrice at 1-minute interval during the procedure. The maximum reading of the diastolic blood pressure was taken as the final value. Then the rise in diastolic blood pressure was calculated by subtracting resting diastolic blood pressure from this value. A rise in DBP of less than $10 \mathrm{~mm} \mathrm{Hg}$ was defined as abnormal, $11-15 \mathrm{~mm} \mathrm{Hg}$ as borderline and $16 \mathrm{~mm} \mathrm{Hg}$ or more as normal. The interpretation of these tests are according to norm adopted by Ewing and Clarke. Heart rate response to deep breathing, valsalva maneuver and standing are known as tests to evaluate parasympathetic nervous system pathway; whereas BP response to standing and to sustained handgrip allow the assessment of sympathetic nervous system activity. The resultsobtained by performing afore-mentioned cardiovascular tests were classified as normal, borderline and abnormal as per the following table. Each individual test was given score of 0,1 , or 2 depending on whether they were respectively normal, borderline or abnormal. An overall autonomic test score of $0-10$ can then be obtained. The data so obtained was entered in a suitable master chart and treated statistically.

Cat-ion exchange resin method to estimate Glycated hemoglobin level:

Glycated hemoglobin: HbA1c levels are estimated by using glycohemoglobin reagent set, provided by RECOMBIGEN LABORATORIES PVT.Ltd 
Table 1 : Cardiovascular autonomic function tests and autonomic score

\begin{tabular}{|l|l|l|l|}
\hline $\begin{array}{l}\text { Heart Rate response tests } \\
\text { (parasympathetic } \\
\text { function) }\end{array}$ & $\begin{array}{l}\text { Normal } \\
\text { (score 0) }\end{array}$ & $\begin{array}{l}\text { Borderline } \\
\text { (score 1) }\end{array}$ & $\begin{array}{l}\text { Abnormal } \\
\text { (score 2) }\end{array}$ \\
\hline $\begin{array}{l}\text { Heart rate response to Valsalva } \\
\text { Maneuver.(VR) }\end{array}$ & 1.21 or more & $1.11-1.20$ & 1.10 or less. \\
\hline $\begin{array}{l}\text { Heart rate response during deep } \\
\text { breathing.(DBD) }\end{array}$ & 15 beats/min & $11-14$ & 10 beats $/ \mathrm{min}$ \\
\hline $\begin{array}{l}\text { Heart rat response to standing.(PTI) } \\
\text { Blood Pressure(BP) Response } \\
\text { tests(sympathetic function) }\end{array}$ & Or more & beats $/ \mathrm{min}$ & or less. \\
\hline BP response to standing & or & $1.01-1.03$ & $1.00 \mathrm{or} \mathrm{less.}$ \\
\cline { 2 - 5 } \\
(fall in systolic BP)
\end{tabular}

\section{Data analysis:}

Data was analyzed by SPSS 20.0.

Results and discussion: Cardiovascular autonomic neuropathy is a most common and earliest complication of chronic hyperglycemia irrespective of the type of diabetes mellitus. Various studies have shown that the degree of cardiac autonomic dysfunction depend on long term glycemic control, duration of diabetes, age, and genetic constitution of the individual, Quantitative autonomic function tests are widely used to assess autonomic function. The Deep breathing test, Valsalva maneuver, Postural tachycardia index, Orthostatic test and Isometric handgrip tests have been used as simple, reliable and reproducible as well as non-invasive methods of studying the cardiac autonomic function. cardiac autonomic dysfunction indicate diffuse damage throughout the Autonomic Nervous system. Against this background, the present study was undertaken to evaluate the effect of glycemic control on cardiovascular autonomic function in poorly controlled diabetes and match the data so obtained in well controlled diabetes and also with healthy controls.

\section{HbA1c in three study groups:}

Mean, SD and CV of HbAlc in the study groups are shown in table. Mean and SD of HbA1c in control, Diabetics with good control, and Diabetics with poor control are $5.8 \pm 0.2,6.5 \pm$ 0.4 and $8.0 \pm 0.8$ respectively. The CV(\%) of control, Diabetics with good control, and Diabetics with poor control are 3.4, 6.1 and 10.0 respectively.

\section{Heart rate response to deep breathing:-}

Heart rate response to deep breathing was evaluated in 90 subjects grouped into control, Diabetics with good control, and Diabetics with poor control. Each group consisting of 30 subjects. Mean, Standard deviation \& Coefficient of variation of Deep breathing difference in the different groups are shown in the table. Mean \& SD of three groups are $17.53 \pm 6.44,11.40 \pm 5.76$ and $7.70 \pm 5.25$ respectively. $\pm \%$. The CV of three groups are $36.57 \%$, $50.0 \%$ and $67.53 \%$ respectively. It is evident from the table that DBD were significantly reduced in Diabetics with good control, and Diabetics with poor control groups when compared with control. DBD $(\mathrm{P}<0.001)$. The DBD was significantly reduced in Diabetics with poor control group compared to DM with good control group showing progressive decline of heart rate response to deep breathing in higher HbAlc level group $(\mathrm{P}<0.001)$. The graphical representation of Mean of Deep breathing difference in the different groups are shown in figure. showing progressive decline in heart rate response to deep breathing. It can thus be concluded from the table $1 \mathrm{a}, 1 \mathrm{~b}, \& 1 \mathrm{c}$ that the heart rate response to deep breathing, a measure of cardiac parasympathetic function is reduced in 1 st, 2nd\& $3 \mathrm{rd}$.group. The basis of finding is multifactorial \& may be at multiple levels of neuraxis including peripheral \& central mechanism as reviewed earlier under effect of glycemic control over cardiac autonomic dysfunction. HR response to breathing is a normal phenomenon and is due primarily to fluctuations in parasympathetic output to heart. During inspiration impulses in vagi from stretch receptors in lungs inhibit the cardio- inhibitory area in medulla oblongata. The 
tonic vagal discharge that keeps the heart rate slow decreases and heart rate rises. In diabetes, loss of vagal tone (vagal denervation) is responsible for reduced heart rate response to deep breathing in diabetes. Parasympathetic fibers being the longest fibers are affected first due to atherosclerotic changes of vasa nervosum. In diabetes, there is a cluster of metabolic and hemodynamic abnormalities, including a disadvantageous lipid profile and altered diurnal blood pressure rhythm. The probable etiology is explained under review of literature. The finding of the present study is in conformity with earlier studies.

Oikawa $\mathrm{N}$ et al, studied Heart rate (HR) variations during deep breathing in 162 healthy subjects and 168 diabetics by use of an instantaneous-HR -change continuous recorder. As indices of HR variations, The Mean of HR during deep breathing and the standard deviation (SD) of the HR were determined. . The $90 \%$ confidence limits were calculated for the normal range and the values below normal range were defined as abnormal. In healthy subjects, the values for each test declined with age and the logtransformed data fitted the linear regression. In diabetics, the incidence of abnormal response were 38\% in the Mean and 19\% in the $\mathrm{SD}$, The Mean was the most sensitive index for the autonomic neuropathy. Mackay JD et al, studied Heart rate (HR) variations using heart rate monitoring in the diagnosis of diabetic autonomic neuropathy, and its value in observing the natural history of this disorder, 287 diabetics aged between 20 and 49 years were studied heart rate variation during deep breathing. Heart rate variation (HRV) on deep breathing proved to be the more sensitive diagnostic index of autonomic neuropathy and was abnormal or borderline in 62 of 64 patients with established autonomic symptoms. Oikawa $\mathrm{N}$ et al, performed Heart rate (HR) variations during deep breathing in 95 diabetics and 38 controls by use of an instantaneous-HR-change continuous recorder. Allsubjects were between 40 and 59 years-old. The mean of HR variation in the diabetics were 9.4 beats $/ \mathrm{min}$. This value was significantly lower than those in the controls ( 20.5 beats $/ \mathrm{min})$. The mean of $\mathrm{HR}$ variation in the diabetics correlated negatively to duration of diabetes and mean fasting blood glucose during the last 6 months in the diabetics, thus, cardiac parasympathetic nerve function was significantly impaired in the diabetics as compared with the controls and that parasympathetic nerve damage occurred early whereas sympathetic innervation was preserved.

\section{Heart rate response to Valsalva Maneuver:}

Heart rate response to Valsalva Maneuver was evaluated in 90 subjects grouped into control, Diabetics with good control, and Diabetics with poor control groups of 30 each. Mean, SD and $\mathrm{CV}(\%)$ of Valsalva ratio (VR) in different groups is shown in table 6. The mean and SD of VR in control, Diabetics with good control, and Diabetics with poor control are $1.2 \pm 0.2,1.2 \pm 0.1$ and $1.1 \pm 0.1$. respectively. The $\mathrm{CV}(\%)$ of three groups are $16.66,8.33$ and 9.09 respectively. The graphical representation of Mean of Valsalva ratio(VR) in the different groups are shown in figure. The heart rate response to Valsalva manoeure was highly significant when compared between groups 1 and $3(\mathrm{P}<0.01 \mathrm{HS})$, significant between groups 2 and $3(\mathrm{P}<0.05 \mathrm{~S})$, but insignificant between groups 1 and $2(\mathrm{P}>0.05 \mathrm{NS})$. The heart rate response to Valsalva maneuver relies to some extent on the integrity of sympathetic as well as parasympathetic pathways. Parasympathetic fibers being the longest fibers are affected first due to atherosclerotic changes of vasa nervosum. In diabetes, changes in metabolic and hemodynamic abnormalities, including a disadvantageous lipid profile, altered diurnal blood pressure rhythm.sd36 The probable etiology is explained under review of literature.The finding of the present study is in conformity with earlier studies.

\section{Heart rate response to standing (PTI):}

Heart rate response to standing was evaluated in 90 subjects grouped into control, Diabetics with good control, and Diabetics with poor control. .The Mean, SD and CV of PTI in different groups is shown in table. The Mean, SD and CV of PTI in control, Diabetics with good control, and Diabetics with poor control are $1.05 \pm 0.10,1.04 \pm 0.06$ and $1.00 \pm 0.07$ respectively. The CV (\%) of control, Diabetics with good control, and Diabetics with poor control are 9.52, 5.76 and 7.00 respectively. The graphical representation of Mean of PTI in the different groups are shown in fig. The decline in PTI was higly significant between

groups 1 and $3(\mathrm{P}<0.01)$ but just significant between groups 1 and 2,2 and 3. $(\mathrm{P}<0.05)$. It can thus be concluded that from tables 9, 10, 11 and fig 10 that the heart rate response to standing, a measure of cardiac parasympathetic function is reduced in groups 2 and 3 compared to controls. The finding of the present study is in conformity with earlier studies. The finding of the present study is in conformity with earlier studies. (supporting studies on decline of dbd).

Oikawa $\mathrm{N}$ et al studied Heart rate (HR) variations--in supine resting position, during deep breathing and on standing-were measured in 162 healthy subjects and 168 diabetics by use of an instantaneous-HR-change continuous recorder. the HR increase on standing (delta HR) was determined. In healthy subjects, the values for each test declined with age and the logtransformed data fitted the linear regression. The $90 \%$ confidence limits were calculated for the normal range and the values below normal range were defined as abnormal. In diabetics, the incidence of abnormal response were $19 \%$ in the SD of HR, 38\% in the delta I-E and $22 \%$ in the delta HR. The delta I-E was the most sensitive index for the autonomic neuropathy. The delta HR was considered to be able to detect the different mechanisms of neural reflexes because of the poor correlation between the delta HR and the respiratory HR variations. The present studies suggested that delta I-E and delta HR should be measured at the same time to evaluate the autonomic neuropathy

\section{Blood Pressure response to standing (Orthostatic test):}

Blood Pressure response to standing was evaluated in 90 subjects grouped into control, Diabetics with good control, and Diabetics with poor control. The Mean, SD and CV of Orthostatic test in different groups is shown in table. The Mean, SD and CV of Orthostatic test in control, Diabetics with good control, and Diabetics with poor control are $12.0 \pm 8.8,9.5 \pm 6.1$ and $18.1 \pm 10.7$ respectively. The CV(\%) of control, Diabetics with good control, and Diabetics with poor control are 73.33, 64.21 and 59.11 respectively. Blood pressure response to standing was significantly reduced in Diabetics. Decrease in SBP on standing from lying was highly significant between groups 1 and 3, groups 2 and $3(\mathrm{P}<0.01)$. while it was not significant between groups 1 and $2(\mathrm{P}>0.05 \mathrm{NS})$. It can thus be concluded that from tables and figure, that the decrease in systolic Blood pressure on standing, a measure of cardiac sympathetic function is increased in both Diabetics with good control, and Diabetics with poor control. The finding of the present study is in conformity with earlier studies.

\section{Blood Pressure response to sustained handgrip:}

Blood Pressure response to sustained handgrip was 
evaluated in 90 subjects grouped into control, Diabetics with good control, and Diabetics with poor control. The Mean, SD and $\mathrm{CV}$ of sustained handgrip in different groups is shown in table. The Mean, SD and CV of sustained handgrip in control, Diabetics with good control, and Diabetics with poor control are $13.1 \pm 6.6,11.3 \pm 7.0$ and $9.8 \pm 6.1$ respectively. The $\mathrm{CV}(\%)$ of control, Diabetics with good control, and Diabetics with poor control are 50.38, 61.94 and 62.24 respectively. The graphical representation of Mean of increased DBP on sustained handgrip in the different groups are shown in fig. Blood pressure response to sustained handgrip was significantly reduced in Diabetics. Increase in DBP tosustained handgrip was highly significant between groups 1 and 2, groups 1 and 3 also groups 2 and 3 ( $\mathrm{P}<0.01$ ). Increase in DBP to sustained handgrip was significantly reduced in both Diabetics with good control, and Diabetics with poor control.

\section{Autonomic Score in three study groups:}

Mean, SD and CV of autonomic score in the study groups are shown in table. Mean and SD of autonomic score in control, Diabetics with good control, and Diabetics with poor control are $4.3 \pm 2.3,4.5 \pm 1.1$ and $6.3 \pm 2.1$ respectively. The $\mathrm{CV}(\%)$ of control, DM without MA, DM with MA are 53.48, 24.44 and 33.33 respectively. The graphical representation of Mean of autonomic scores in the different groups are shown in figure. There was a significant increase in autonomic scores in Diabetics with good control, and Diabetics with poor control when compared to control as seen in table. Autonomic scores was significantly increased in both Diabetics with good control, and Diabetics with poor control $(\mathrm{P}<0.01)$.

The basis of finding is multifactorial and may be at multiple levels of neuraxis including peripheral and central mechanisms as reviewed under pathophysiology of diabetic autonomic neuropathy.Relation between $\mathrm{HbA} 1 \mathrm{c}$ and Autonomic

\section{Score in three study groups:}

The Mean HbA1c of three study groups were 5.8,6.5 and 8.0 respectively, while the mean autonomic scores of three study groups were 3.3, 4.5 and 6.3 respectively. Multiple Comparison of Relation between Mean HbA1c and mean autonomic Score in three study groups were found to be highly significant.

\section{CONCLUSION:}

The basis of finding is multifactorial and may be at multiple levels of neuraxis including peripheral and central mechanisms as reviewed under pathophysiology of diabetic autonomic neuropathy.

\section{REFERENCES}

1. Bell JI, Hockaday TDR. Diabetes mellitus. Oxford Text book of Medicine. 3rd edition. Vol 2:1996:1448-1504.

2. Sztajzel J. Heart rate variability: a noninvasive electrocardiographic method to measure the autonomic nervous system. Swiss Med Wkly 2004; 134:514-522.

3. Vinik AI, Freeman R, Erbas T. Diabetic autonomic neuropathy. Semin Neurol 2003;23:365-372.

4. Ziegler D. Cardiovascular autonomic neuropathy: clinical manifestations and measurement. Diabetes Reviews 1999;7:342-357.

5. Burgos LG, Ebert TJ, Asiddao C, et al. Increased intraoperative cardiovascular morbidity in diabetics with autonomic neuropathy. Anesthesiology 1989;70:591-597.

6. Knuttgen D, Buttner-Belz U, Gernot A, Doehn M. Unstable blood pressure during anesthesia in diabetic patients with autonomic neuropathy. Anasth Intensivther Notfallmed 1990;25:256-262.

7. Latson TW, Ashmore TH, Reinhart DJ, Klein KW, Giesecke AH. Autonomic reflex dysfunction in patients presenting for elective surgery is associated with hypotension after anesthesia induction. Anesthesiology 1994;80:326-337.

8. Purewal TS, Watkins PJ. Postural hypotension in diabetic autonomic neuropathy: a review. Diabet Med 1995;12:192-200.

9. Pfeifer MA. Cardiovascular autonomic neuropathy: advances in testing help unlock its complexity. Diabetes Spectrum 1990; 3:45-48.

10. Shakespeare CF, Katritsis D, Crowther A, Cooper IC, Coltart JD, Webb-Peploe MM. Differences in autonomic nerve function in patients with silent and symptomatic myocardial ischemia. Br Heart J 1994;71:22-29.

11. Piha SJ. Cardiovascular autonomic function tests. Responses in healthy subjects and determination of age related reference value. Rehab research centre 1988;1148.

12. Hirsch JA, Bishop B. Respiratory sinus arrhythmia in humans: how breathing pattern modulates heart rate. Am J Physiol 1981;241:H620-H629.

13. Levin AB. A Simple test of cardiac function based upon the heart changes induced by valsalva maneuver. Am J Cardiol 1966;18:90-99.

14. Ewing DJ, Hume L, Campbell IW, et al. Autonomic mechanism in initial heart rate response to standing. J Appl Physiol 1980;49:809-814.

15. Piha SJ. Cardiovascular responses to various autonomic tests in males and females. Clin Auton Res 1993;3(1):1520.

16. Piha SJ. Cardiovascular autonomic reflex tests: Normal responses and age related reference values. Clin Physiol 1991;11:277-290

17. Vita G. Cardiovascular reflex tests: Assessment of ageadjusted normal range. J Neural Sci 1986;75(3):263-74.

18. Goldstein DE, Little RR, Lorenz RA, Malone JI, Nathan D, Peterson CM, Sacks DB. Diabetes care 2003; 27:1761-73.).

19. DCCT. The effect of intensive treatment of diabetes on the development and progression of long-term complications in insulin- dependent diabetes mellitus.N Engl j Med 1993; 329:977-86.

20. U.K.Prospective Study (UKPDS) Group. Intensive blood-glucose control with Sulphonylureas or insulin compared with conventional treatment and risk of complications in patients with type 2 diabetes (UKPDS). UK Prospective Study (UKPDS) Group. 33 lancet 1998; $352: 837-53$ 